\title{
High-density linkage map construction and QTL analysis for earliness-related traits in Gossypium hirsutum L
}

\author{
Xiaoyun Jia ${ }^{1,2}$, Chaoyou Pang ${ }^{2}$, Hengling Wei ${ }^{2}$, Hantao Wang ${ }^{2}$, Qifeng $\mathrm{Ma}^{2}$, Jilong Yang ${ }^{2}$, Shuaishuai Cheng ${ }^{1,2}$, \\ Junji Su ${ }^{1,2}$, Shuli Fan ${ }^{2}$, Meizhen Song ${ }^{2}$, Nusireti Wusiman ${ }^{3}$ and Shuxun $\mathrm{Yu}^{1,2^{*}}$
}

\begin{abstract}
Background: Gossypium hirsutum L., or upland cotton, is an important renewable resource for textile fiber. To enhance understanding of the genetic basis of cotton earliness, we constructed an intra-specific recombinant inbred line population (RIL) containing 137 lines, and performed linkage map construction and quantitative trait locus (QTL) mapping.

Results: Using restriction-site associated DNA sequencing, a genetic map composed of 6,434 loci, including 6,295 single nucleotide polymorphisms and 139 simple sequence repeat loci, was developed from RIL population. This map spanned 4,071.98 cM, with an average distance of $0.63 \mathrm{cM}$ between adjacent markers. A total of 247 QTLs for six earliness-related traits were detected in 6 consecutive years. In addition, 55 QTL coincidence regions representing more than $60 \%$ of total QTLs were found on 22 chromosomes, which indicated that several earliness-related traits might be simultaneously improved. Fine-mapping of a 2-Mb region on chromosome D3 associated with five stable QTLs between Marker25958 and Marker25963 revealed that lines containing alleles derived from CCRI36 in this region exhibited smaller phenotypes and earlier maturity. One candidate gene (EMF2) was predicted and validated by quantitative real-time PCR in early-, medium- and late-maturing cultivars from 3- to 6-leaf stages, with highest expression level in early-maturing cultivar, CCRI74, lowest expression level in late-maturing cultivar, Bomian1.

Conclusions: We developed an SNP-based genetic map, and this map is the first high-density genetic map for short-season cotton and has the potential to provide deeper insights into earliness. Cotton earliness-related QTLs and QTL coincidence regions will provide useful materials for QTL fine mapping, gene positional cloning and MAS. And the gene, EMF2, is promising for further study.
\end{abstract}

Keywords: Gossypium hirsutum L, Cotton earliness, Single nucleotide polymorphism (SNP), High-density genetic map, Quantitative trait loci (QTLS)

\section{Background}

Gossypium hirsutum L. $(2 n=4 x=52)$, one of 50 Gossypium species and the leading natural fiber crop, contributes to more than $95 \%$ of total cotton production $[1,2]$. Short-season cotton, or early-maturity cotton, is a type of G. hirsutum with a relatively short growth period. Earliness, a distinctive characteristic of short-season cotton, is a complex agronomic trait of a quantitative

\footnotetext{
* Correspondence: ysx195311@163.com

${ }^{1}$ College of Agronomy, Northwest A\&F University, Yangling 712100, China

${ }^{2}$ State Key Laboratory of Cotton Biology, Institute of Cotton Research of

CAAS, Anyang 455000, China

Full list of author information is available at the end of the article
}

genetic nature [3]. In terms of plant development, earliness may be described in reference to budding date, flowering timing (FT) and the whole growth period (WGP) [4]. Other agronomic traits, such as plant height $(\mathrm{PH})$, node of the first fruiting branch (NFFB) and height of the NFFB (HNFFB), have also been used as earliness indexes [5, 6]. For example, Godoy and Palomo concluded that the lower the NFFB and the shorter the PH, the earlier the maturity [5]. To alleviate food crises, a large amount of farmland previously used for cotton planting is now devoted to food crops, especially in China. To exploit these limited natural resources via 
breeding programs and manage the competition between cereal crops and cotton through crop rotation, cotton earliness is attracting an increasing amount of attention from breeders [6].

Although a decrease in genetic diversity increases the difficulty of breeding by hybridization [7], marker-assisted selection (MAS) is a promising approach for cotton breeding because it shortens breeding cycles with more accuracy [8]. Following the publication of the first allotetraploid cotton genetic map in 1994 [1], a host of research programs have been dedicated to genetic map construction [9-15] and quantitative trait locus (QTL) mapping [16-19]. Because anonymous marker-based maps are becoming saturated, a genetic map based on conventional markers cannot achieve the resolution required for fine QTL mapping or positional cloning. Single nucleotide polymorphisms (SNPs) are highly abundant and suitable for high-density genetic mapping [20, 21]. Restriction-site associated DNA sequencing (RAD-seq) is a powerful and cost-effective method for detecting genetic variations $[22,23]$ and has become widely applied in plants [24-28]. Moreover, landmark breakthroughs in genome sequencing of cotton species such as G. raimondii [29, 30] G. arboretum [31], G. barbadense [32, 33] and G. hirsutum L. [34, 35], have been achieved and will facilitate the detection of SNP markers.

Compared with the number of studies evaluating fiber quality, limited investigations have been conducted to identify genetic signatures for earliness [36-43]. The genetic bases of earliness are poorly understood and present findings are insufficient for application to breeding practices. To provide additional information for breeding programs designed for specific traits, the molecular mechanisms underlying cotton earliness must thus be further investigated.

The research strengthes of our team in regard to short-season cotton were exploited to advance genetic understanding of earliness, thereby allowing us to construct immortalized recombinant inbred lines (RILs) consisting of 137 families from two G. hirsutum inbred lines: CCRI36 and G2005. The purpose of the present study was to (1) construct an intra-specific highresolution genetic map for G. hirsutum using RAD-seq, (2) detect QTLs related to earliness for MAS in shortseason cotton breeding and (3) lay a foundation for fine mapping and positional cloning for use in future studies.

\section{Results}

SNP detection and genetic map construction

For library construction, Taqd ${ }^{\mathrm{I}}$ was chosen as the enzyme mainly because of its uniformly distributed cutting sites and sufficient coverage across the entire cotton genome (Additional file 1). We obtained 14,109,670 and 18,558,010 paired-end reads for CCRI36 and G2005, respectively, and 7,293,849 paired-end reads per line for the RIL population. The average Q30 score was 86.44\%, demonstrating the high quality of the sequences (Table 1). All read sequences generated in this study are available in the Sequence Read Archive (http://www.ncbi.nlm.nih.gov/ Traces/sra/) under accession number PRJNA315785. For the genome sequence alignment of G. hirsutum [35], the depth of the parental materials reached $10.57 \times$ for G2005 and $6.43 \times$ for CCRI36. The average sequence depth of the 137 lines reached 4.59× (Table 1). We obtained 369,223 SNPs, of which 78,127 were polymorphic between the parents. The At sub-genome contained 227,397 SNPs, of which 43,869 (19.29\%) were polymorphic; the Dt subgenome contained 141,826 SNPs, of which 34,258 $(24.16 \%)$ were polymorphic (Additional file 2 ). The percentage of marker polymorphisms on each chromosome varied from $3.4 \%$ on chromosome A10 to $36.85 \%$ on A6.

For the construction of the genetic map, simple SNPs between the parents, represented as aa $\times b b$, were selected in addition to the SSR markers. The final genetic map was composed of 6,434 loci including 6,295 simple SNPs and 139 SSR loci; it spanned 4,071.98 cM, with an average distance of $0.63 \mathrm{cM}$ between adjacent markers (Fig. 1, Table 2, and Additional file 3). The At subgenome contained a larger number of markers $(3,536$ SNPs and 64 SSR loci) compared with the Dt subgenome (2,759 SNPs and 75 SSR loci). The average distance between adjacent markers was $0.57 \mathrm{cM}$ in the At sub-genome and $0.71 \mathrm{cM}$ in the Dt sub-genome. Marker distributions and linkage lengths varied among chromosomes. Chromosome A6 contained the most loci, 411, whereas D11 contained the fewest, 112. The largest linkage group was A9, with 196.04 cM; the shortest was D4, with $98.98 \mathrm{cM}$. The highest marker density was on A6, which had an average marker interval of only $0.36 \mathrm{cM}$. The largest gap among all chromosomes was 18.24 cM on D10.

Table 1 Statistical description of sequence data obtained from the parents and the recombinant inbred line (RIL) population

\begin{tabular}{lccccc}
\hline Sample & Total Reads (M) & Average Q30(\%) & Average GC content (\%) & Average depth $(\times)$ & Coverage $(\%)$ \\
\hline CCRI36 & 14.11 & 89.75 & 40.74 & 6.43 & 8.17 \\
G2005 & 18.56 & 90.95 & 39.88 & 10.57 & 9.16 \\
RILs & 999.26 & 86.38 & 40.22 & 4.59 & 7.09 \\
\hline
\end{tabular}

Q30 is a base call error rate of 0.001 


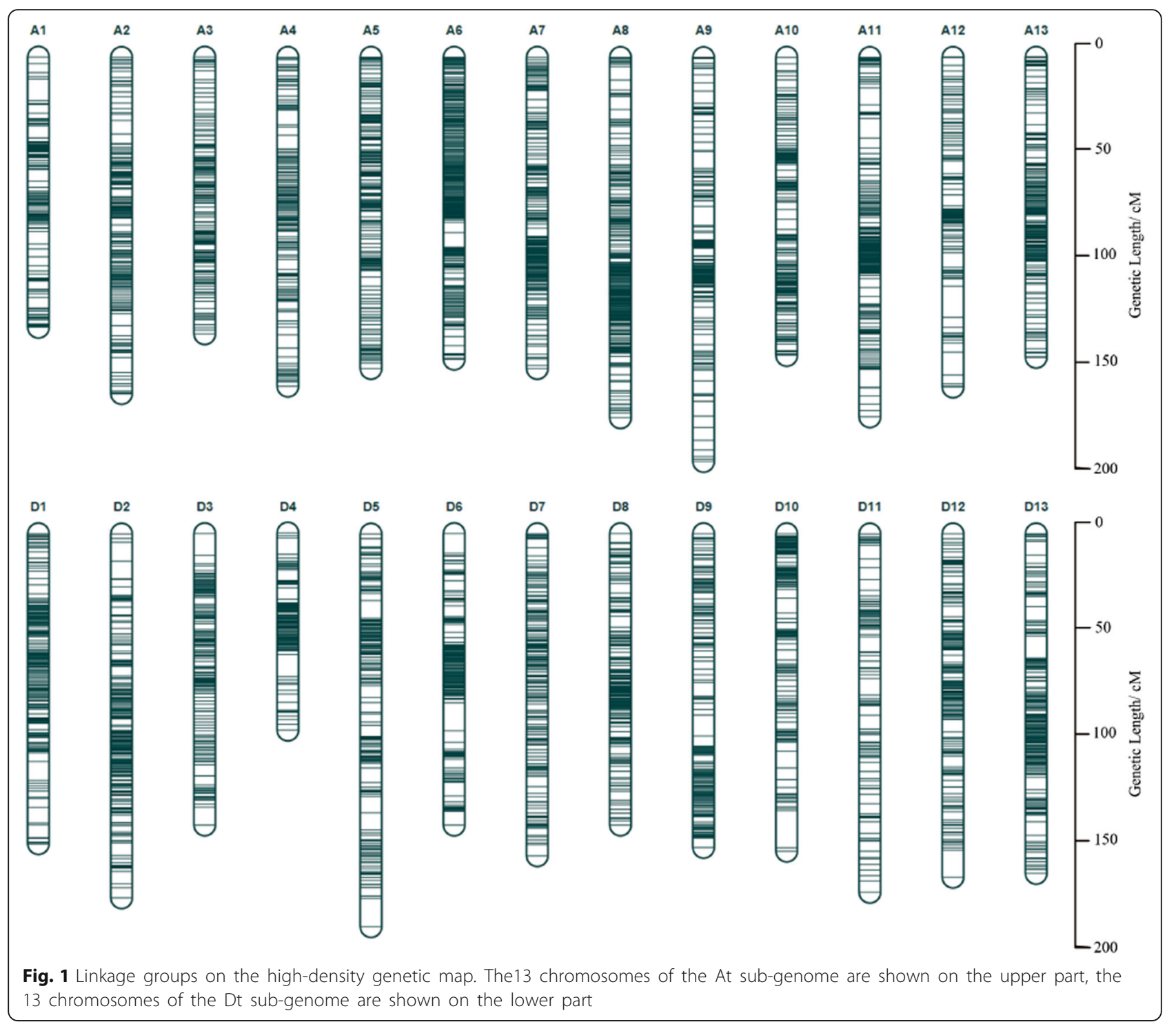

\section{Phenotypic variation}

The mapping population was planted continuously from 2010 to 2015 at Anyang. We surveyed six earlinessrelated traits: WGP, FT, FBP, PH, NFFB and HNFFB.

Large differences existed between the parents, with G2005 exhibiting a later FT and NFFB appearance, a longer WGP and FBP, and a higher $\mathrm{PH}$ and HNFFB than CCRI36 (Additional file 4). All the six traits followed an approximately normal distribution and showed transgressive segregation in the RIL population, which indicated that the latter was a typical population for QTL detection (Additional file 4). Although been significantly affected by environmental effects, the broad-sense heritabilities of the six traits ranged from $74.93 \%$ to $85.13 \%$, thereby demonstrating that the phenotypic variation was mainly genetically controlled and indicating in turn the potential for performing genetic modifications for earliness (Table 3). A simple correlation analysis showed that all six traits were positively correlated with one another (Additional file 5). FT was significantly positively correlated with the other five traits in all 6 years except with NFFB in 2011 and FBP in 2014 and 2015, in which significance levels of $p=0.05$ or $p=0.01$ were not reached. WGP was also significantly positively correlated with the other five traits in the 6 years except for with HNFFB in 2014. PH had a significant positive correlation with the other five traits except for with FBP in 2010 and 2014. FBP showed significant positive correlations with WGP and FT over the 6 years, with less significant correlations with PH, NFFB or HNFFB in 2010 and 2014. NFFB was significantly positively correlated with the other five traits over the years except for 2011; for this year, only the correlation between NFFB and HNFFB reached the $p=0.05$ significance level. HNFFB was significantly positively correlated with the other five 
Table 2 Detailed information on the single nucleotide polymorphism (SNP)-based genetic map

\begin{tabular}{|c|c|c|c|c|c|c|}
\hline Chromosome & Loci & SNP loci & SSR loci & Length (cM) & Average interval (cM) & largest gap (cM) \\
\hline $\mathrm{A} 1$ & 190 & 184 & 6 & 133.2 & 0.7 & 10.611 \\
\hline$A 2$ & 218 & 216 & 2 & 163.92 & 0.76 & 7.254 \\
\hline A3 & 218 & 212 & 6 & 136.23 & 0.63 & 4.376 \\
\hline A4 & 169 & 169 & 0 & 160.85 & 0.95 & 7.19 \\
\hline A5 & 325 & 325 & 0 & 152.42 & 0.47 & 4.41 \\
\hline A6 & 411 & 399 & 12 & 147.89 & 0.36 & 7.192 \\
\hline A7 & 305 & 305 & 0 & 152.55 & 0.5 & 4.38 \\
\hline A8 & 386 & 386 & 0 & 175.59 & 0.45 & 6.65 \\
\hline A9 & 281 & 261 & 20 & 196.04 & 0.7 & 9.152 \\
\hline $\mathrm{A} 10$ & 258 & 258 & 0 & 146.32 & 0.57 & 4.89 \\
\hline A11 & 357 & 349 & 8 & 174.97 & 0.49 & 9.685 \\
\hline $\mathrm{A} 12$ & 157 & 154 & 3 & 160.88 & 1.03 & 15.37 \\
\hline $\mathrm{A} 13$ & 325 & 318 & 7 & 147.25 & 0.45 & 5.763 \\
\hline At sub-genome & 3600 & 3536 & 64 & 2048.11 & 0.57 & 15.37 \\
\hline D1 & 257 & 240 & 17 & 152.01 & 0.59 & 9.246 \\
\hline D2 & 268 & 258 & 10 & 177.07 & 0.66 & 9.311 \\
\hline D3 & 179 & 179 & 0 & 143.18 & 0.8 & 6.65 \\
\hline D4 & 178 & 178 & 0 & 98.98 & 0.56 & 11.19 \\
\hline D5 & 252 & 241 & 11 & 190.57 & 0.76 & 13.703 \\
\hline D6 & 258 & 241 & 17 & 143.48 & 0.56 & 13.763 \\
\hline D7 & 208 & 208 & 0 & 157.77 & 0.76 & 5.48 \\
\hline D8 & 257 & 257 & 0 & 143.18 & 0.56 & 5.3 \\
\hline D9 & 201 & 189 & 12 & 153.85 & 0.77 & 10.321 \\
\hline D10 & 198 & 193 & 5 & 155.65 & 0.79 & 18.24 \\
\hline D11 & 112 & 109 & 3 & 174.54 & 1.57 & 8.071 \\
\hline D12 & 229 & 229 & 0 & 167.8 & 0.73 & 13.15 \\
\hline D13 & 237 & 237 & 0 & 165.79 & 0.7 & 10.72 \\
\hline Dt sub-genome & 2834 & 2759 & 75 & 2023.87 & 0.71 & 18.24 \\
\hline Summary & 6434 & 6295 & 139 & 4071.98 & 0.63 & 18.24 \\
\hline
\end{tabular}

traits in the 6 years except for WGP and FBP in 2014. The results of the correlation analysis are consistent with the conclusion that the lower the NFFB and the shorter the PH, the earlier the maturity [5], and also suggest the high likelihood of gene pleiotropy or tightly linked functional genes.

\section{QTL analysis}

To reveal the genetic factors underlying cotton earliness, data for the six traits for the 6 years were analyzed separately and then combined using average values. A total of 247 QTLs were identified on all 26 chromosomes,

Table 3 ANOVA analysis of the six earliness-related traits

\begin{tabular}{|c|c|c|c|c|c|c|}
\hline Source of variation & $\mathrm{FT}$ & WGP & FBP & $\mathrm{PH}$ & HNFFB & NFFB \\
\hline $\mathrm{G}$ & $56.11^{* * *}$ & $293.87^{* * *}$ & $197.16^{* * *}$ & $314.98^{* * *}$ & $73.64^{* * *}$ & $5.76^{* * *}$ \\
\hline E & $11081.84^{* * *}$ & $7202.56^{* * *}$ & $6488.76^{* * *}$ & $79418.15^{* * *}$ & $14480.68^{* * *}$ & $55.03^{* * *}$ \\
\hline$G \times E$ & $9.43^{* * *}$ & $84.36^{* * *}$ & $68.92^{* * *}$ & $56.21^{* *}$ & $14.18^{* * *}$ & $0.68^{* * *}$ \\
\hline error & 3.37 & 20.93 & 18.76 & 44.27 & 8.98 & 0.27 \\
\hline
\end{tabular}

G Genotype, E Environment, $F T$ flowering timing, WGP whole growth period, FBP flowering to boll-opening period, $P H$ plant height, NFFB node of the first fruiting branch, HNFFB height of the NFFB

** significant at the $p<0.01$; *** significant at the $p<0.001$ 
including 39 QTLs for FT, 47 for WGP, 40 for FBP, 33 for PH, 43 for NFFB, and 45 for HNFFB (Additional file 6). Chromosome D3 contained 30 QTLs and ranked first among the 26 chromosomes, whereas A1, A6, D2, D6 and D11 contained fewer than 5 QTLs. A total of 52 stable QTLs were detected in at least two environments, and 52 QTLs accounted for more than $10 \%$ of the phenotypic variation (PV). The paternal material G2005 and maternal material CCRI36 provided 146 and 101 of the trait-increasing alleles, respectively. Of the total QTLs, 109 were located on the At sub-genome and 138 were located on the Dt sub-genome. Nineteen stable QTLs were located on the At sub-genome, with the remaining 33 on the Dt sub-genome.

\section{WGP}

WGP is important for successful cultivation and represents the first index for evaluating earliness because it directly indicates the extent of maturity [5]. Forty-seven QTLs for WGP were identified, including 10 stable and 6 major QTLs. Of the three stable QTLs located on D3, qGP-D3-4 was detected in 3 years and in the combined analysis. qWGP-D3-2 at $96.91 \mathrm{cM}$ was detected in 2011 and 2012 and explained $12.33 \%-18.27 \%$ of the PV. qWGP-A12-1, detected in 2015 and in the combined analysis, respectively explained $15.24 \%$ and $10.12 \%$ of the observed PV.

\section{FT}

Flowering is the transition from vegetative to reproductive stages and represents an efficient signal of earliness [44, 45]. For maximal yield, tailoring of FT in the crop life cycle is essential. In this study, a total of 39 QTLs were detected for FT, with 5 explaining $11.73 \%-29.37 \%$ of the PV and 6 being stably detected over time. qFT-D3-3 at 95.61-96.91 cM was stably detected in five years from 2011 to 2015 and in the combined analysis; it accounted for $19.14 \%, 29.37 \%$, $21.31 \%$ and $22.97 \%$ of the PV in 2011, 2012, 2013 and the combined analysis, respectively. qFT-D3-2 at 86.81$87.11 \mathrm{cM}$ was detected in 3 years and in the combined analysis and explained $25.99 \%$ and $19.91 \%$ of the PV in 2012 and the combined analysis, respectively. Application of FT as an indicator of cotton earliness revealed the fragment from 86.87-96.91 cM on D3 as an interesting candidate region, with CCRI36 conferred favorable alleles.

\section{FBP}

FBP is a primary component of cotton development after flowering and directly affects harvesting rate. Forty QTLs were detected for FBP, with 10 stable QTLs detected in at least 2 environments and 9 explaining more than $10 \%$ of the PV. The four QTLs, qFBP-A5-1, qFBP-D3-2, qFBP-D51 and qFBP-D7-3, were detected in three environments; the latter two QTLs explained more than $10 \%$ of the PV. The
QTL qFBP-D13-2 was detected in 2013 and 2015 and explained more than $10 \%$ of the PV in both years.

\section{$\mathrm{PH}$}

$\mathrm{PH}$ is an important agronomic trait that influences plant architecture, production and mechanization. Thirty-three QTLs for PH were obtained, including 11 stable QTLs and 9 major QTLs that explained $10 \%-21.77 \%$ of the observed PV. Eight of the 11 stable QTLs were detected in the Dt sub-genome, and 6 were detected on chromosome D3. The five QTLs, qPH-D3-2 (at $57.11 \mathrm{cM}$ ), qPHD3-3 (62.81-64.81 cM), qPH-D3-4 (83.81-87.11 cM), qPH-D3-5 (95.61-96.91 cM) and qPH-D12-3 (100.41$101.51 \mathrm{cM}$ ), were detected in three environments, with G2005 conferring the favorable allele increasing PH. qPHD3-2 and qPH-D3-3, detected in 2013 and 2015 and in the combined analysis, explained $10.85 \%-21.77 \%$ and $7.51 \%-14.96 \%$ of the PV, respectively. qPH-D3-4 and qPH-D3-5 were detected in 2011, 2012 and 2014, and explained $6.03 \%-11.27 \%$ and $6.74 \%-14.66 \%$ of the PV, respectively. qPH-D12-3 was detected in 2013-2015, and explained $4.29 \%-9.09 \%$ of the PV.

\section{NFFB}

NFFB has been suggested as an indicator of FT and a measure of relative photoperiodism [39]. A total of 43 QTLs were detected for NFFB in this study, with 12 major QTLs explaining $10.30 \%-23.71 \%$ of the PV. All nine stable QTLs were in the Dt sub-genome. qNFFB-D2-4 (at 134.11$134.91 \mathrm{cM})$ and qNFFB-D3-2 (96.91-101.91 cM) were detected in 3 years and in the combined analysis; they explained $6.59 \%-13.10 \%$ and $6.33 \%-15.63 \%$ of the PV, respectively. Another seven stable QTLs were detected in two environments.

\section{HNFFB}

HNFFB, a primary component of $\mathrm{PH}$, is significantly positively correlated with FT and WGP. Forty-five QTLs for HNFFB were detected, including 6 stable and 11 major QTLs. The four stable QTLs, qHNFFB-A4-1, qHNFFB-A7-1, qHNFFB-A11-3 and qHNFFB-A13-4, were located on the At sub-genome. Two notable QTLs, qHNFFB-D3-6 at 86.81-90.51 cM and qHNFFB-D3-7 at $96.91 \mathrm{cM}$, were detected in 2011, 2012 and 2015 and in the combined analysis; they explained $13.88 \%-20.04 \%$ and $17.03 \%-24.71 \%$ of the PV, respectively. G2005 conferred the favorable allele that increased HNFFB.

\section{QTL coincidence and gene annotation}

The QTL mapping results indicated 55 QTL coincidence regions (with $95 \%$ confidence intervals overlapped) on 22 chromosomes, and more than $60 \%$ of all QTLs (158/247; $64.37 \%$ ) were involved in these regions. A total of $70.21 \%$ of the WGP QTLs-the highest value among the six 
traits-were located in coincidence regions, followed by QTLs for HNFFB (68.89\%), FBP (67.50\%), PH (63.64\%), NFFB (62.79\%) and FT (51.28 \%). Most QTLs in the same coincidence regions presented the same allelic direction, a phenomenon that explains the significant positive correlation among the six traits and is consistent with the speculation of gene pleiotropy or tightly linked functional genes (Table 4, Additional file 7). In addition, each trait had characteristic QTLs that might be useful for specific trait modification or ideotype breeding.

FT and FBP are two of the main components of WGP. FT and WGP had 10 common QTL coincidence regions that included 11 FT and 13 WGP QTLs, whereas FBP and WGP had 20 common coincidence regions that included 22 FBP and 22 WGP QTLs. In contrast, only six common coincidence regions were identified between FT and FBP; they included 7 FT and 7 FBP QTLs. HNFFB is a primary component of $\mathrm{PH}$, and they shared 13 common QTL coincidence regions that included 17 HNFFB and 15 PH QTLs (Table 5). This phenomenon demonstrates the plasticity of earliness-related traits during developmental stages.

Among the 55 QTL coincidence regions, one chromosome region on D3 was extraordinary. Five stable QTLs were located in the core interval of 95.61-96.91 cM: qFTD3-3, qWGP-D3-2, qPH-D3-5, qNFFB-D3-2 and qHN FFB-D3-7, which all had the same allele direction. In addition, qFBP-D3-1, which was detected in 2011, was also found in this region (Fig. 2, Additional file 8). Among the six QTLs, qFT-D3-3 was stably detected from 2011 to 2015 and was also detected in the combined analysis; thus, this QTL features high reliability for fine mapping. Six SNPs were mapped in the core region defined by Marker25957 and Marker25965. Based on the homozygous genotype of these six SNPs, we classified the 137 RILs into two groups. As expected, the lines inheriting the homozygous QTLs from CCRI36 flowered and matured earlier and had smaller phenotypes than those carrying the homozygous allele of G2005; in addition, all six traits exhibited stable phenotypic differences among the 6 years

Table 4 Quantitative trait loci (QTL) for the six studied traits and QTLS located in coincidence regions

\begin{tabular}{lllc}
\hline Trait & Total QTL & Coincided QTL & Percentage (\%) \\
\hline FT & 39 & 20 & 51.28 \\
FBP & 40 & 27 & 67.50 \\
WGP & 47 & 33 & 70.21 \\
NFFB & 43 & 26 & 62.79 \\
HNFFB & 45 & 31 & 68.89 \\
PH & 33 & 21 & 63.64 \\
total & 247 & 159 & 64.37 \\
\hline
\end{tabular}

$F T$ flowering timing, WGP whole growth period, $F B P$ flowering to boll-opening period, $P H$ plant height, NFFB node of the first fruiting branch, HNFFB height of the NFFB
(Fig. 3). A total of 12 recombinants occurred in the RIL population at this locus, which allowed us to narrow down the interval to a 2-Mb region between Marker25958 and Marker25963 (Additional file 9). A total of 68 genes were annotated in this region (Additional file 10).

One gene, named $E M F 2$, was primarily verified by qRTPCR. The qRT-PCR analysis reflected that EMF2 had the lowest expression level in the early-maturing cultivar CCRI74 and the highest in Bomian1, with Shan70 intermediate (Additional file 11). In all three cultivars, the expression level of $E M F 2$ decreased during the progression from the three- to the six true-leaf stage. This EMF2 expression trend was consistent with that of flower buds differentiation in cotton. We therefore conclude that EMF2 in cotton may also have a significant influence on flowering and is thus a potential candidate gene for use in further studies.

\section{Discussion}

In this study, we selected CCRI36 and G2005 as parents for construction of a RIL population containing 137 lines. CCRI36 is an excellent early-maturity cultivar with high yield production. G2005 is a G. barbadense introgression line in the background of G. hirsutum and has a relatively longer growth period. This hybrid combination can both enrich the diversity of the intra-specific genetic mapping population and introduce desirable genes from G. barbadense into upland cotton for direct use on breeding, thus overcoming the difficulties of inter-specific crossing in cotton breeding [9]. The generated RIL population is therefore of great significance, both for theoretical genetic research and elite germplasm selection.

Genetic map is the first requisite for understanding the genetic mechanisms of a target trait. Rong et al. constructed a genetic map including 3,347 loci with $1.78 \mathrm{cM}$ between adjacent markers [10]. Yu et al. published a genetic map with 2,316 loci and $1.91 \mathrm{cM}$ in average marker distance [12]. Yu et al. reported a genetic map containing 2,072 loci with an average marker interval of $1.63 \mathrm{cM}$ [13]. Liang et al. reported an updated map consisting of 3,414 loci with an average marker interval of $1.08 \mathrm{cM}$ [14]. Shi et al. constructed a genetic map with 2,292 loci and $2.23 \mathrm{cM}$ between adjacent markers [15]. Previously released genetic maps of Gossypium have primarily been based on PCRbased markers, which are laborious, time consuming and gradually less effective because of saturation during highdensity genetic map construction. Since the application of high-throughput sequencing on cotton, marker density has been significantly increased. Wang et al. constructed an ultra-dense genetic map consisting of 4,999,048 SNPs distributed across 4,042 cM, and these authors used their high-density genetic map for genome assembly of Gossypium hirsutum acc. TM-1 [21]. Wang et al. applied the RAD-seq technique to upland cotton and identified 21,109 SNPs based on the genome sequence of G. hirsutum TM-1. 
Table 5 Number of coincidence regions and coincident quantitative trait loci (QTLs) between 15 trait pairs

\begin{tabular}{|c|c|c|c|c|c|c|}
\hline Trait & FT & FBP & WGP & NFFB & HNFFB & $\mathrm{PH}$ \\
\hline FT & & & 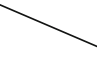 & 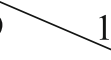 & & \\
\hline FBP & $6^{a}$ & & & & & \\
\hline WGP & 10 & 20 & & & & \\
\hline NFFB & 8 & 7 & 10 & & 17 & \\
\hline HNFFB & 8 & 7 & 6 & 13 & & \\
\hline $\mathrm{PH}$ & 4 & 3 & 4 & 5 & 13 & \\
\hline
\end{tabular}

FT flowering timing, WGP whole growth period, FBP flowering to boll-opening period, PH plant height, NFFB node of the first fruiting branch, HNFFB height of the NFFB

${ }^{a}$ number of coincident $\mathrm{QTLs}$ between FT and FBP, ${ }^{b}$ number of QTLs for FT in coincidence regions between FT and FBP, ${ }^{c}$, number of QTLs number for FBP in coincidence regions between FT and FBP

They constructed a high-density genetic map comprising 3,984 RAD markers and 169 SSR markers and encompassing 3,500 cM, with $0.84 \mathrm{cM}$ between adjacent markers [27]. Hulse-Kemp et al. constructed an ultra-high density genetic map using SNP arrays for marker detection and constructed a high-density genetic map consisting of 19,191 markers with $0.21 \mathrm{cM}$ between adjacent markers [46]. Zhang et al. constructed a high-density genetic map that contained 5,521 SNPs with an average marker density of $0.78 \mathrm{cM}$ [47]. Thus, high-throughput sequencing techniques are more efficient.

To construct a satisfactory high-density genetic map for short-season cotton, we adopted RAD-seq technology and developed 78,127 polymorphic SNPs between the parents. Polymorphisms between the parents reached $21.16 \%$, which was much higher than the value for SSR markers (Additional file 2). The genetic map, which contained 6,295 simple SNPs and 139 SSR loci, spanned 4,071.98 cM with an average of $0.63 \mathrm{cM}$ between adjacent markers; this corresponds to a map length comparable to previous maps and a better marker density (Fig. 1, Table 2, Additional file $3)$. The mapped markers were uniformly distributed along the 26 cotton chromosomes (Additional file 12). The quality of our genetic map was evaluated by heat map analysis, which confirmed the accuracy of the 26 linkage groups because the recombination frequency among adjacent markers was relatively low (Additional file 13). In addition, linkage information for the 139 SSR loci was validated by a comparison with genetic maps reported by Liang et al. [14] and Blenda et al. [48]; in particular, the distribution of loci in their maps confirmed the common-marker-based assignment of the 26 linkage groups in our study
(Additional file 14). Most of the marker collinearity between the genetic map and the genome sequence was clear, thus illustrating that the markers were accurately located on the present genetic map and had sufficient coverage on the 26 chromosomes (Fig. 4). Our map is the first highdensity genetic map for short-season cotton based on SNP markers. In combination with the other published genetic maps described above, our map represents one of the most valuable resources available for cotton genome detection.

Published genetic studies on cotton have given priority to fiber quality [49], whereas few studies have focused on earliness. In this study, based on the high-density genetic map, we conducted QTL mapping for cotton earliness. The six studied traits were positively correlated with one another, which indicates the existence of gene pleiotropy or closely linked functional genes. This finding is consistent with the observation that more than half of detected QTLs (158/247; $64.37 \%$ ) were involved in QTL coincidence regions and that QTLs in the same coincidence region were characterized by the same allelic direction (Table 4, Additional file 4, Additional file 7); this phenomenon reveals the possibility of synchronously improving several earliness-related traits. FT and FBP are two of the main components of the WGP; however, the QTLs for these traits only partially coincided or overlapped. The same result was found between HNFFB and $\mathrm{PH}$, with HNFFB is a primarily component of $\mathrm{PH}$ (Table 5). These limited common coincidence regions indicate that these genes might be expressed constitutively throughout the whole growth period or within the whole plant. In addition, various genes likely function at different developmental stages or in different parts of the plant, with each stage or organ modifiable by the ectopic expression of 
D3

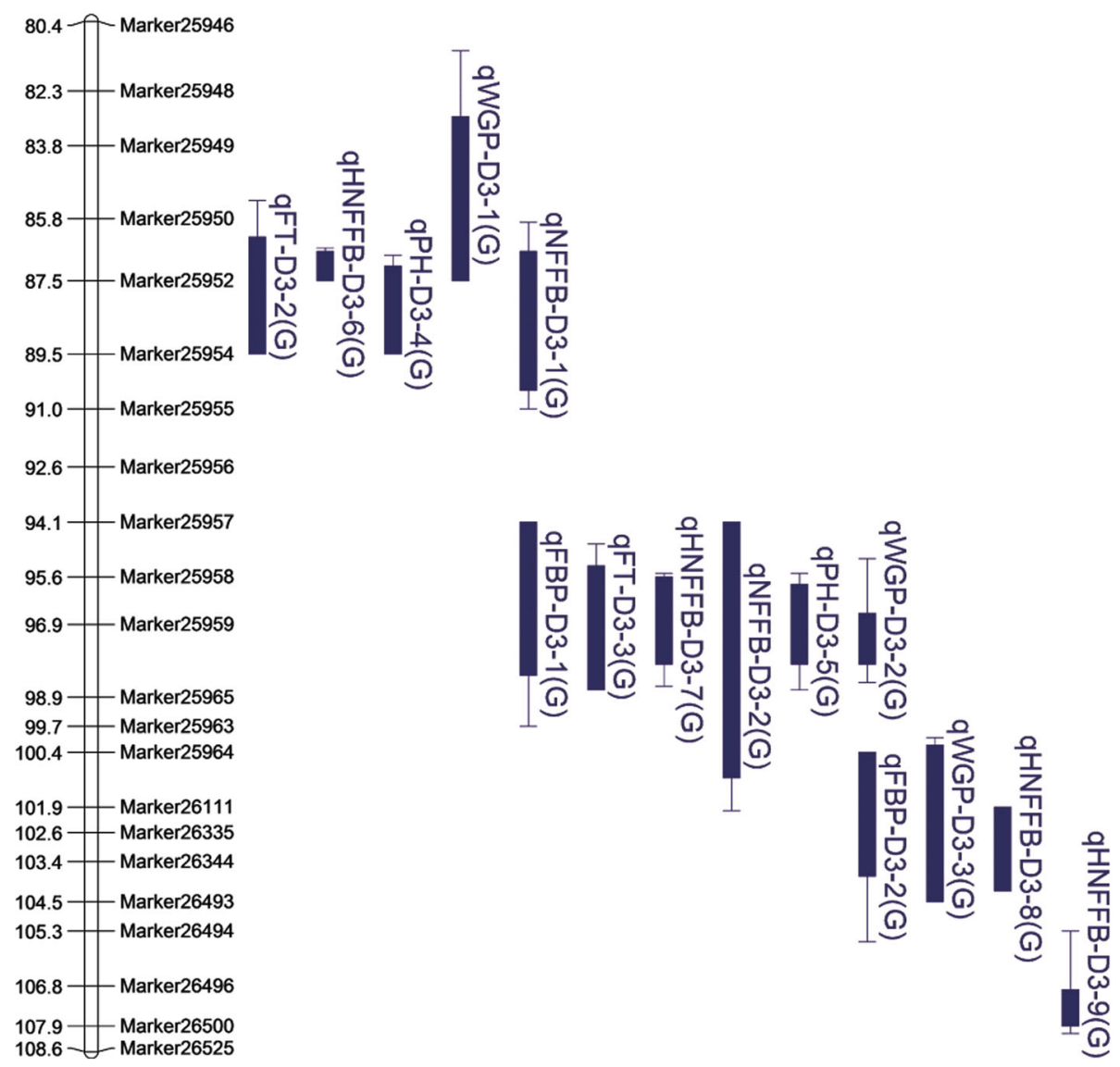

Fig. 2 Quantitative trait loci detected on a noteworthy region of chromosome D3. FBP: flowering to boll-opening period; PH: plant height; FT: flowering timing; WGP: whole growth period; NFFB: node of the first fruiting branch; HNFFB: height of the NFFB

specific genes. Cotton earliness can therefore be specifically improved. And the needs of plants at different growth stages and environments must further be considered to support the most appropriate conditions for plant development.

Previous studies detected earliness-related QTLs distributed on almost all 26 cotton chromosomes [36-43]. In particular, QTLs for FBP, WGP, NFFB, HNFFB and PH were detected as follows: QTLs for FBP on chromosomes A3, A7, A8/D8, A9, A13, D3, D4 and D7 [38]; QTLs for WGP on chromosomes A13, D3 and D8 [38]; QTLs for NFFB on chromosomes A1, A5, A6, A9, A10, A11, A13, D1, D3, D6, D7, D9 and D11 [37, 39, 41]; QTLs for HNFFB on chromosomes A9, D3 and D6 [37]; and QTLs for PH on chromosomes A3, A4, A5, A9, A11, A13, D2, D3, D4, D5, D6, D8, D9 and D11 $[36,42]$. These results indicate that D3 contains QTLs for FBP, WGP, NFFB, HNFFB and PH, which suggests that D3 is one of the chromosomes responsible for cotton earliness. Because of limitations in marker density and useful genome information, however, the published QTLs with large confidence intervals are not well understood and additional studies had not been reported prior to the present investigation. By analyzing the high-density genetic map, we identified more than 200 QTLs, including 30 QTLs on D3. A stable QTL at $95.61 \mathrm{cM}$, qFT-D3-3, was identified in six environments and coincided with another five QTLs in the interval of 95.61-96.91 cM. This result demonstrates that this region may contain a critical gene(s) tightly related to earliness (Fig. 2). Several other QTLs were also detected upstream or downstream of this region (Fig. 2, Additional file 8). Homozygous RILs in this interval were clearly classifiable into two groups with different phenotypes. The first group, which inherited homozygous QTL alleles from the G2005 cultivar, exhibited larger plant architectures and later flowering. The other group, which inherited alleles from the CCRI36 cultivar, was characterized by smaller phenotypes and earlier flowering (Fig. 3). On the basis of 12 recombinants in the RIL population, we narrowed down the core interval to a region spanning approximately $2 \mathrm{Mb}$ between Marker25958 and Marker25963 (Additional file 9). Sixty-eight genes were annotated in this interval; one of them, $E M F 2$, was regularly expressed among cultivars with different growth periods (Additional 


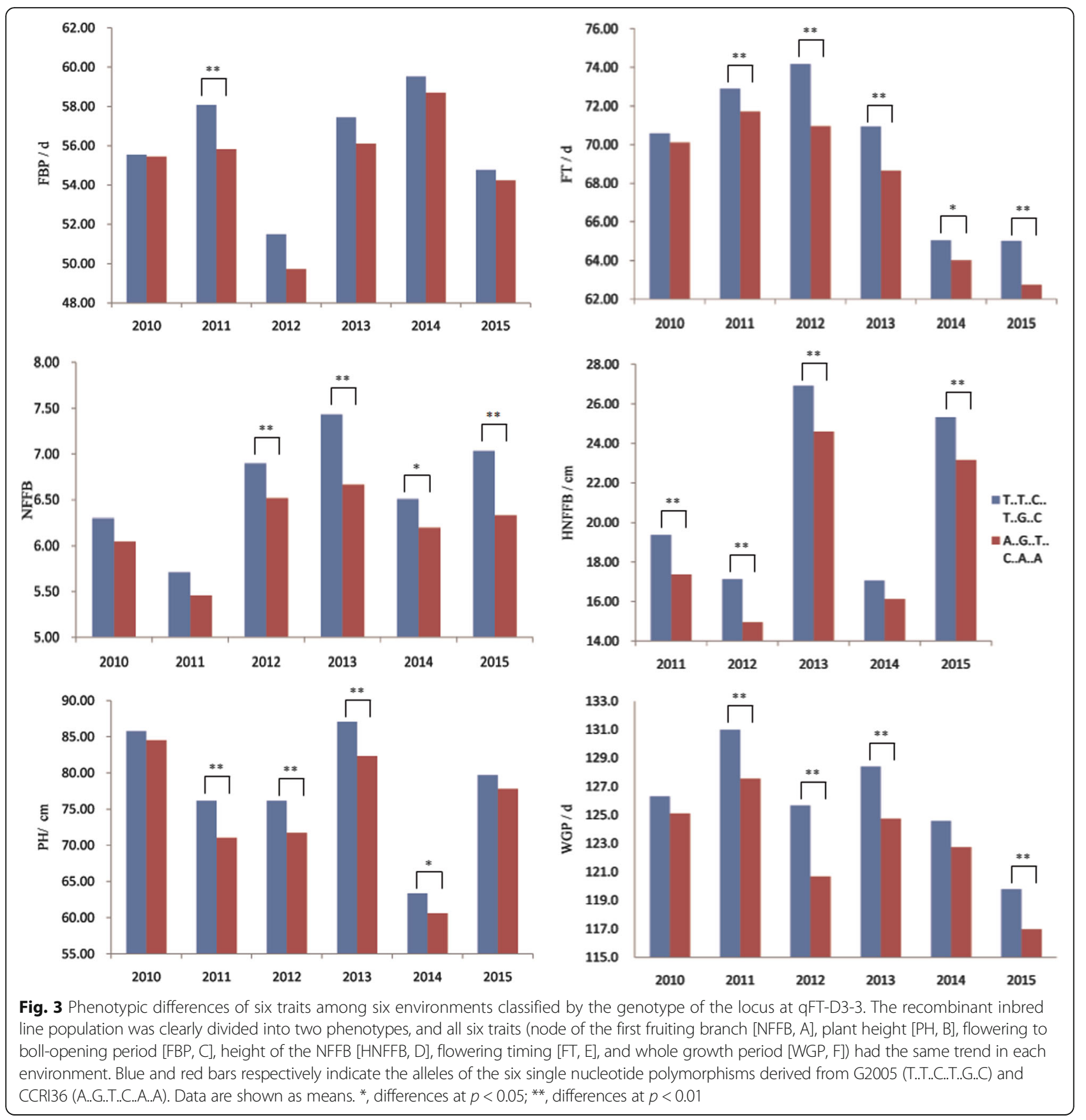

file 10). At all four stages, EMF2 displayed relatively low expression in CCRI74 compared with Shan70, but showed the highest expression in Bomian1 (Additional file 11). Earlier studies found that EMF2 is involved in various pathways, including delayed flowering [50-52]. We speculate that $E M F 2$ in cotton also has a major role in flowering. Because of limitations related to population type or size, however, further pinpointing the candidate interval based on the 137-RIL population will be difficult. New populations are consequently needed, such as near isogenic lines (NILs) and larger secondary $F_{2}$ populations. And we plan to perform additional work involving on QTL fine mapping and functional verifications in future studies.

\section{Conclusions}

In this study, we developed a high-density genetic map, the first SNP-based map constructed for short-season cotton that will be useful for genetic studies of earliness. More than 200 QTLs were detected and 55 QTL coincidence regions were analyzed; these results will provide useful materials for QTL fine mapping, gene positional cloning and MAS. One gene, EMF2, was verified by 


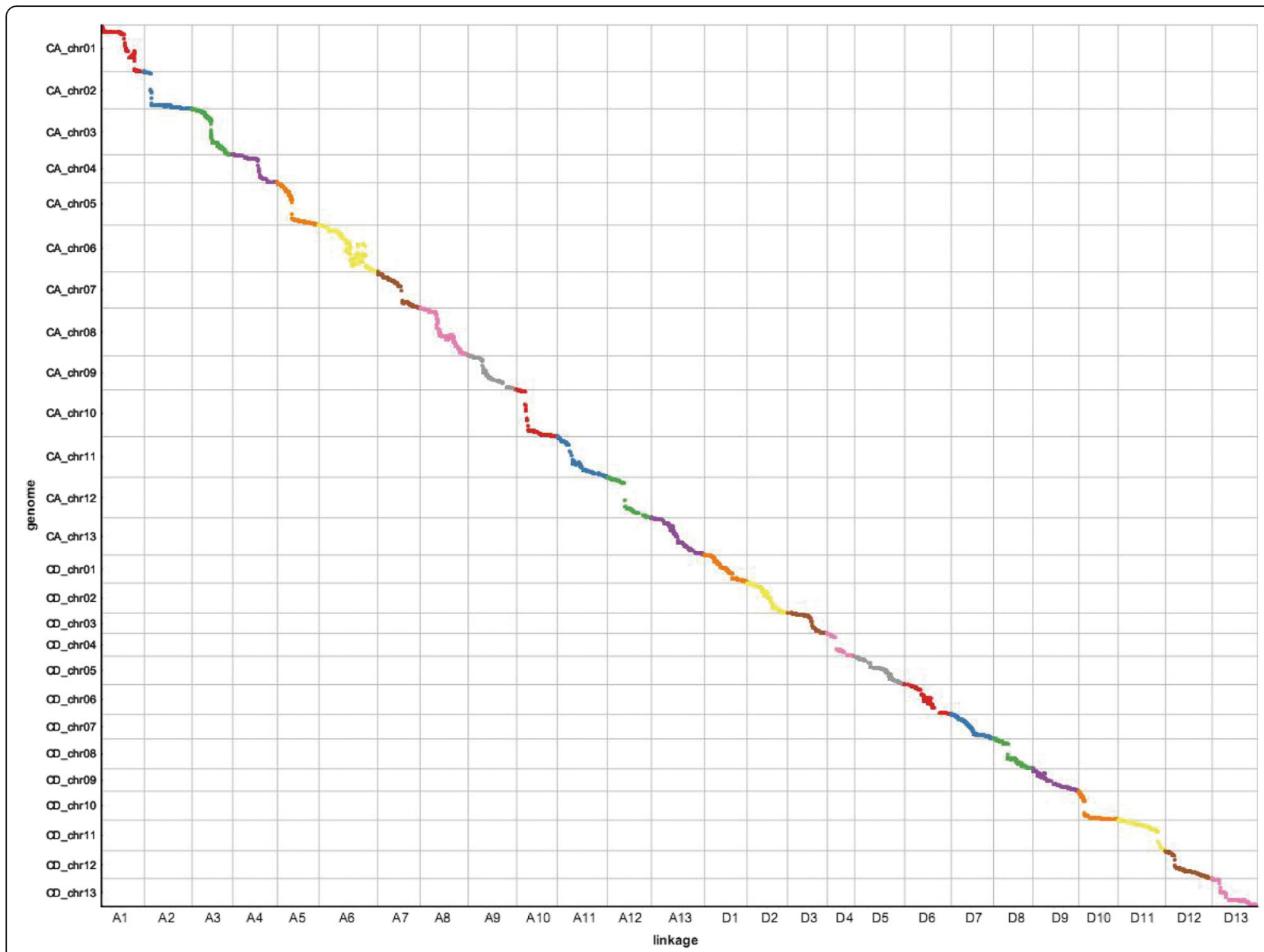

Fig. 4 Marker collinearity between the genetic map and the reference genome. The $x$-axis indicates the arrangement of the 26 linkage groups $(\mathrm{cM})$; the $\mathrm{y}$-axis indicates the 26 reference chromosome sequences (Mb)

qRT-PCR among three cultivars from 3- to 6-leaf developmental stages; the result demonstrated this gene is promising for further study. In addition, the approach used in this study, RAD-seq is a powerful technique for SNP detection in high-resolution genetic map construction with polyploidy species.

\section{Methods}

\section{Mapping population and statistical analysis}

Gossypium hirsutum accession CCRI36 and an introgression line from G. barbadense G2005 were used as parental materials in a cross in 2006. CCRI36 and G2005 are upland cotton cultivars/lines bred by our lab. $\mathrm{F}_{1}$ seeds were planted in Hainan, China, in the winter of 2006. A total of $137 \mathrm{~F}_{2}$ plants were randomly selected and self-mated at Anyang, Henan province, China, in 2007. Beginning from the $F_{2: 3}$ generation, single-seed descent was conducted until the $\mathrm{F}_{2: 9}$ generation to construct a RIL population. All RILs and parents were planted on the farm of the Cotton Research Institute of the Chinese Academy of Agricultural
Sciences at Anyang from 2010 to 2015. A randomized complete block design was adopted with three replicates per year, with 25 plants retained in 5-m-long rows spaced $80 \mathrm{~cm}$ apart. We surveyed six earliness-related traits: $\mathrm{PH}$ (height from the ground to the main stem tip), NFFB (node number of the first fruiting branch, with the cotyledonary node recorded as zero), HNFFB (height from the ground to the NFFB), WGP (the number of days from the date of sowing to the date when $50 \%$ of plants reached boll opening), FT (the number of days from the date of sowing to the date when $50 \%$ of plants were flowering), and flowering to boll-opening period (FBP, the number of days from flowering to boll opening). All of the plants were used for the WGP, FT and FBP surveys, whereas 10 plants in the middle of each row were selected for the NFFB, HNFFB and PH measurements.

Phenotypic data were analyzed using SAS software (SAS Institute, Inc., Cary, NC, USA). The PROC GLM procedure in SAS was used to estimate the variance. An analysis of variance was performed for the yearly data, and the results 
over 6 years were combined. Broad-sense heritability was calculated according to Knapp et al. [53].

Three cultivars were used for gene validation: CCRI74, Shan70 and Bomian1. CCRI74 is an early-maturing cultivar with a 105-day WGP, Bomian1 is a late-maturing cultivar with a 135-day WGP, and Shan70 is an intermediatematuring cultivar with a 117-day WGP.

\section{DNA extraction and simple sequence repeat (SSR) analysis}

Genomic DNA was extracted from young leaves of the 137-RIL population and the two parents using the CTAB method [54]. A total of 3,624 SSR primer pairs were previously screened for polymorphisms between the parents; of these, 152 polymorphic primers were used to genotype the RIL population. All primer sequences used in this study can be downloaded from CottonGen (https://www.cottongen.org/). The G2005 genotype was designed as A, the CCRI36 genotype was designed as B and the heterozygous genotype was designed as $\mathrm{H}$. If an SSR primer amplified more than one polymorphic band, a suffix of a/b/c was added after the primer name according to the product length from longest to shortest.

\section{Bar-coded library preparation}

Bar-coded libraries were created as described by Andolfatto et al. [55] with several modifications, with 6-bp barcoded adapters designed and modified according to the standard Illumina adapter protocol for paired-end read libraries. DNA was enzyme digested using FastDigest Taqa ${ }^{\mathrm{I}}$ (Thermo scientific Fermentas) and adapter ligation was carried out with T4 DNA ligase (Enzymatics). Subsequently, 19 to 24 of the resulting samples with different indexes were pooled together. DNA fragments between 400 and 600 bp were retrieved and purified using a QIAquick Gel Extraction kit (Qiagen, Valencia, CA). After purification, the adapterligated DNA fractions were subjected to PCR amplification (Phusion High-fidelity, Finnzymes). The PCR products were gel separated, and the 400-600-bp DNA fractions were purified using a QIAquick PCR Purification kit (Qiagen, Valencia, CA). Finally, the purified libraries were quantified on an Agilent 2100 Bioanalyzer and sequenced on an Illumina HiSeq 2000 instrument at BGI, Shenzhen, China.

\section{Sequence alignment and SNP identification}

Genomic libraries were prepared and sequenced on the Illumina HiSeq 2000 platform according to the manufacturer's instructions. Reads from multiple Illumina sequence channels were first filtered and segregated with the appropriate adapters assigned to each sample. Sequence alignment and SNP discovery were realized using the method described by Chen et al. [56]. All qualified sequences were mapped back to genome sequences of G. hirsutum [35] using the short-read sequences alignment software program
BWA (version 0.5.9-r16) [57]. The Genome Analysis Toolkit (GATK) was used as the best practical approach for variation detection, with SNPs that were common between SAMTools and GATK considered to be candidates $[58,59]$. Given the RIL population, SNPs that were homozygous in both parents were used to construct the genetic map. We selected single-dose SNPs that were only present on one homologous chromosome.

\section{Map construction and QTL analysis}

Polymorphic SNPs were refiltered prior to map construction. SNPs were removed if they met any of the following five criteria: 1) the SNP was missing in more than $20 \%$ of RILs; 2$)$ the $p$-value of the segregation distortion was less than 0.01 ; 3) the sequencing depth of the parents was less than $4 \times ; 4$ ) either of the parents was heterozygous (heterozygosity $>20 \%$ ); and 5) the SNPs were redundant and in the same reads. Map construction was performed with a HighMap strategy as detailed in Liu [60]. The input datasets included the SSR marker and SNP genotypes. First, the high-quality markers were clustered into linkage groups based on a pair-wise modified logarithm of the odds (LOD) score for the recombination frequency. Second, Gibbs sampling, spatial sampling, and a simulated annealing algorithm were used to order the markers and estimate map distances. Third, genotyping errors (singletons) were identified and eliminated according to parental contributions to the genotypes using the k-nearest neighbor algorithm. To order the markers correctly, ordering and error correction processes were conducted iteratively. After several cycles, high-density linkage maps were obtained. Finally, heat maps were constructed and SNP collinearity was assessed to evaluate the map quality. The Kosambi function was used to convert the recombination frequencies into centimorgans.

For the QTL analysis, phenotypic data from 6 years were used separately and in combination. The combined analysis was conducted using the average of the phenotypic data. The QTLs were analyzed by composite interval mapping using WinQTLCart 2.5. The parameters were set to $5 \mathrm{cM}$ of the window size and $1 \mathrm{cM}$ of the walk speed, with up to 10 background markers. A LOD score $\geq 2.5$ was used to detect QTLs. QTLs at the same location for the same trait across different years or in the same environments were regarded as 'stable', with QTLs explaining more than $10 \%$ of the phenotypic variation (PV) regarded as 'major'. QTLs were named according to McCouch et al. [61].

Gene annotation and quantitative real-time PCR (qRT-PCR) Genes in the target region were annotated using the annotation information reported by Tianzhen Zhang [35]. Total RNA was extracted from the apical buds of CCRI74, Shan70 and Bomian1 cultivars at three- to six-leaf stages using an RNAprep Pure Kit for Plants (Tiangen, China). First-strand cDNA synthesis was performed on $2 \mu \mathrm{g}$ of 
total RNA using a First Strand cDNA Synthesis kit (Toyobo, Japan). The synthesized cDNA samples were diluted 10-fold and then used as CDNA templates for the qRT-PCR analysis. The qRT-PCR analysis was conducted using an Applied Biosystems 7500 Fast Real-Time PCR System and Fast SYBR Green Master Mix (Life Technologies, Foster City, CA, USA) with three technical replicates.

\section{Additional files}

Additional file 1: Distribution of the Taqal enzyme recognition site on the 26 cotton chromosomes. (TIF $1211 \mathrm{~kb}$ )

Additional file 2: Genotypes of the 6,295 polymorphic SNPs between the parents and SNP polymorphisms on each chromosome. (XLSX $221 \mathrm{~kb}$ )

Additional file 3: Detailed marker positions and linkage length of the 26 linkage groups. (XLSX $143 \mathrm{~kb}$ )

Additional file 4: Phenotypic distribution for the six traits of the parents and the RIL population. FBP: flowering to boll-opening period; PH: plant height; FT: flowering timing; WGP: whole growth period; NFFB: node of the first fruiting branch; HNFFB: height of the NFFB. (XLS $30 \mathrm{~kb}$ )

Additional file 5: Correlation coefficient ( $r$ ) matrices for the six traits measured in the RIL population. FBP: flowering to boll-opening period; PH: plant height; FT: flowering timing; WGP: whole growth period; NFFB: node of the first fruiting branch; HNFFB: height of the NFFB. (XLS $29 \mathrm{~kb}$ )

Additional file 6: Detailed information of the 247 QTLs for the six cotton earliness-related traits from the RIL population. Stable or major QTLs are marked in yellow. (XLS $84 \mathrm{~kb}$ )

Additional file 7: QTL and QTL coincidence region distributions on the genetic map. QTLs located in the coincidence regions are marked in blue. (PDF $343 \mathrm{~kb}$ )

Additional file 8: The LOD score of QTLs coinciding with GFT-D3-3 on chromosome D3. FBP: flowering to boll-opening period; PH: plant height; FT: flowering timing; WGP: whole growth period; NFFB: node of the first fruiting branch; HNFFB: height of the NFFB. (TIF $3549 \mathrm{~kb}$ )

Additional file 9: Recombinants in the RIL population at locus qFT-D3-3 defining the core region between Marker25957 and Marker25965. CCRI36 is the maternal cultivar; G2005 is the paternal line; $b$ is the homozygous allele conferred by CCRI36; $a$ is the homozygous allele conferred by G2005; and $\mathrm{h}$ is heterozygous. (PDF $124 \mathrm{~kb}$ )

Additional file 10: Genes annotated between Marker25958 and Marker25963. (XLS $65 \mathrm{~kb})$

Additional file 11: EMF2 expression levels in apical buds of CCRI74, Shan70 and Bomian1. RNA was extracted during three- to six-true-leaf developmental stages. CCRI74, Shan70 and Bomian1 are respectively early-, intermediate- and late-maturing cultivars. Data are represented as means. (TIF $82 \mathrm{~kb}$ )

Additional file 12: Distribution of the mapped SNPs on the 26 reference chromosomes. The horizontal line indicates the physical position (Mb) of the 26 chromosomes, and the vertical line indicates the 26 chromosomes from D13 to A1. (TIF $1351 \mathrm{~kb}$ )

Additional file 13: Heat maps of the 26 linkage groups. (PDF $4231 \mathrm{~kb}$ ) Additional file 14: SSR locus positions on the genetic map and common markers between our map and the references. (XLS $40 \mathrm{~kb}$ )

\section{Abbreviations}

FBP: Flowering to boll-opening period; FT: Flowering timing; HNFFB: Height of NFFB; NFFB: Node of first fruiting branch; PH: Plant height; PV: Phenotypic variation; qRT-PCR: Quantitative real-time PCR; QTL: Quantitative trait locus; RAD-seq: Restriction-site associated DNA sequencing; RIL: Recombinant inbred line; SNP: Single nucleotide polymorphism; WGP: Whole growth period

\section{Acknowledgements}

The experiment was performed at the State Key Laboratory of Cotton Biology at the Institute of Cotton Research of the Chinese Academy of Agricultural Sciences. This work was funded by the China Agriculture Research System (No. CARS-18) and the National Natural Fund Project (No. 31360352).

\section{Availability of supporting data and materials}

Sequences are available on the Sequence Read Archive http://www.ncbi. nIm.nih.gov/Traces/sra/ under accession number PRJNA315785. And the data sets supporting the results of this study are included in the manuscript and its Additional files 1, 2, 3, 4, 5, 6, 7, 8, 9, 10, 11, 12, 13 and 14 .

\section{Authors' contributions}

SY, SF and MS designed the experiment; XJ, JY and CP performed the data collection; HW and QM analyzed the sequence data; XJ, JS and HW analyzed the phenotypic data; JY, NW and XJ performed the SSR experiment; SC conducted the QTL analysis and GRT-PCR analysis; and XJ wrote and revised the manuscript. All of the authors read and approved the final manuscript.

\section{Competing interests}

The authors declare that they have no competing interests.

\section{Consent for publication}

Not applicable

Ethics approval and consent to participate Not applicable

\section{Author details}

${ }^{1}$ College of Agronomy, Northwest A\&F University, Yangling 712100, China. ${ }^{2}$ State Key Laboratory of Cotton Biology, Institute of Cotton Research of CAAS, Anyang 455000, China. ${ }^{3}$ Institute of Industrial Crops of Xinjiang Academy of Agricultural Sciences, Xinjiang 830091, China.

Received: 13 July 2016 Accepted: 5 November 2016

Published online: 11 November 2016

\section{References}

1. Reinisch AJ, Dong JM, Brubaker CL, Stelly DM, Wendel JF, Paterson AH. A detailed RFLP map of cotton, Gossypium hirsutum x Gossypium barbadense: chromosome organization and evolution in a disomic polyploid genome. Genetics. 1994;138:829-47.

2. Chen ZJ, Scheffler BE, Dennis E. Toward sequencing cotton (Gossypium) Genomes. Plant Physiol. 2007;145:1303-10.

3. Yu SX, Huang ZM. Inheritance analysis on earliness components of short season cotton varieties in G. hirsutum. Sci Agric Sin. 1990;23:48-54.

4. Richmond TR, Radwan SRH. A comparative study of seven methods of measuring earliness of crop maturity in cotton. Crop Sci. 1962;2:397-400.

5. Godoy AS, Palomo GA. Genetic analysis of earliness in upland cotton (Gossypium hirsutum L.). I. morphological and phenological variables. Euphytica. 1999;105:155-60.

6. Basbag S, Ekinci R, Gencer O. Combining ability and heterosis for earliness characters in line $x$ tester population of Gossypium hirsutum L. Hereditas. 2007;144:185-90.

7. Frelichowski JE, Palmer MB, Main D, Tomkins JP, Cantrell RG, Stelly DM, Yu J, Kohel RJ, Ulloa M. Cotton genome mapping with new microsatellites from Acala 'Maxxa' BAC-ends. Mol Genet Genomics. 2006:275:479-91.

8. Cao ZB, Wang P, Zhu XF, Chen H, Zhang TZ. SSR marker-assisted improvement of fiber qualities in Gossypium hirsutum using G. barbadense introgression lines. Theor Appl Genet. 2014;127:587-94.

9. Lacape JM, Jacobs J, Arioli T, Derijcker R, Forestier-Chiron N, Llewellyn D, Jean J, Thomas E, Viot C. A new interspecific, Gossypium hirsutum x G. barbadense, RIL population: towards a unified consensus linkage map of tetraploid cotton. Theor Appl Genet. 2009;1 19:281-92.

10. Rong JK, Abbey C, Bowers JE, Brubaker CL, Chang C, Chee PW, Delmonte TA, Ding XL, Garza JJ, Marler BS, et al. A 3347-locus genetic recombination map of sequence-tagged sites reveals features of genome organization, transmission and evolution of cotton (Gossypium). Genetics. 2004;166: 389-417. 
11. Guo WZ, Cai CP, Wang CB, Zhao L, Wang L, Zhang TZ. A preliminary analysis of genome structure and composition in Gossypium hirsutum. BMC Genomics. 2008;9:314.

12. Yu Y, Yuan DJ, Liang SG, Li XM, Wang XQ, Lin ZX, Zhang XL. Genome structure of cotton revealed by a genome-wide SSR genetic map constructed from a BC1 population between Gossypium hirsutum and G. barbadense. BMC Genomics. 2011;12:15.

13. Yu JZ, Kohel RJ, Fang DD, Cho JM, Van Deynze A, Ulloa M, Hoffman SM, Pepper AE, Stelly DM, Jenkins JN, et al. A high-density simple sequence repeat and single nucleotide polymorphism genetic map of the tetraploid cotton genome. G3 (Bethesda). 2012;2:43-58.

14. Liang Z, Lv YD, Cai CP, Tong XC, Chen XD, Zhang W, Du H, Guo XH, Guo WZ. Toward allotetraploid cotton genome assembly: integration of a highdensity molecular genetic linkage map with DNA sequence information. BMC Genomics. 2012;13:539.

15. Shi Y, Li W, Li A, Ge R, Zhang B, Li J, Liu G, Liu A, Shang H, Gong J, et al. Constructing a high-density linkage map for Gossypium hirsutum $\mathrm{x}$ Gossypium barbadense and identifying QTLs for lint percentage. J Integr Plant Biol. 2014:57:450-67.

16. Yu JW, Zhang K, Li SY, Yu SX, Zhai HH, Wu M, Li XL, Fan SL, Song MZ, Yang $D G$, et al. Mapping quantitative trait loci for lint yield and fiber quality across environments in a Gossypium hirsutum x Gossypium barbadense backcross inbred line population. Theor Appl Genet. 2013;126:275-87.

17. Fang DD, Jenkins JN, Deng DD, McCarty JC, Li P, Wu JX. Quantitative trait loci analysis of fiber quality traits using a random-mated recombinant inbred population in upland cotton (Gossypium hirsutum L.). BMC Genomics. 2014;15:397.

18. Tan ZY, Fang XM, Tang SY, Zhang J, Liu DJ, Teng ZH, Li L, Ni HJ, Zheng FM, Liu DX, et al. Genetic map and QTL controlling fiber quality traits in upland cotton (Gossypium hirsutum L.). Euphytica. 2015;203:615-28.

19. Jamshed M, Jia F, Gong J, Palanga KK, Shi Y, Li J, Shang H, Liu A, Chen $T$, Zhang $Z$, et al. Identification of stable quantitative trait loci (QTLs) for fiber quality traits across multiple environments in Gossypium hirsutum recombinant inbred line population. BMC Genomics. 2016;17:197.

20. Kaur S, Kimber RBE, Cogan NOI, Materne M, Forster JW, Paull JG. SNP discovery and high-density genetic mapping in faba bean (Vicia faba L.) permits identification of QTLs for ascochyta blight resistance. Plant Sci. 2014; 217:47-55.

21. Wang S, Chen JD, Zhang WP, Hu Y, Chang LJ, Fang L, Wang Q, Lv FN, Wu $H T$, Si ZF, et al. Sequence-based ultra-dense genetic and physical maps reveal structural variations of allopolyploid cotton genomes. Genome Biol. 2015;16:108.

22. Miller MR, Dunham JP, Amores A, Cresko WA, Johnson EA. Rapid and costeffective polymorphism identification and genotyping using restriction site associated DNA (RAD) markers. Genome Res. 2007:17:240-8.

23. Andrews KR, Good JM, Miller MR, Luikart G, Hohenlohe PA. Harnessing the power of RADseq for ecological and evolutionary genomics. Nat Rev Genet. 2016;17:81-92.

24. Xu P, Xu SZ, Wu XH, Tao Y, Wang BG, Wang S, Qin DH, Lu ZF, Li GJ. Population genomic analyses from low-coverage RAD-Seq data: a case study on the non-model cucurbit bottle gourd. Plant J. 2014;77:430-42.

25. Hegarty M, Yadav R, Lee M, Armstead I, Sanderson R, Scollan N, Powell W, Skot L. Genotyping by RAD sequencing enables mapping of fatty acid composition traits in perennial ryegrass (Lolium perenne (L.)). Plant Biotechnol J. 2013;11:572-81.

26. Wang HT, Jin X, Zhang BB, Shen C, Lin ZX. Enrichment of an intraspecific genetic map of upland cotton by developing markers using parental RAD sequencing. DNA Res. 2015;22:147-60.

27. Wang Y, Ning Z, Hu Y, Chen J, Zhao R, Chen H, Ai N, Guo W, Zhang T. Molecular mapping of restriction-site associated DNA markers in allotetraploid upland cotton. Plos One. 2015;10:e0124781.

28. Chutimanitsakun Y, Nipper RW, Cuesta-Marcos A, Cistue L, Corey A, Filichkina T, Johnson EA, Hayes PM. Construction and application for QTL analysis of a Restriction Site Associated DNA (RAD) linkage map in barley. BMC Genomics. 2011;12:4.

29. Paterson AH, Wendel JF, Gundlach H, Guo H, Jenkins J, Jin DC, Llewellyn D, Showmaker KC, Shu SQ, Udall J, et al. Repeated polyploidization of Gossypium genomes and the evolution of spinnable cotton fibres. Nature. 2012;492:423-7.

30. Wang KB, Wang ZW, Li FG, Ye WW, Wang JY, Song GL, Yue Z, Cong L, Shang HH, Zhu SL, et al. The draft genome of a diploid cotton Gossypium raimondii. Nat Genet. 2012;44:1098-3.
31. Li F, Fan G, Wang K, Sun F, Yuan Y, Song G, Li Q, Ma Z, Lu C, Zou C, et al. Genome sequence of the cultivated cotton Gossypium arboreum. Nat Genet. 2014;46:567-72

32. Liu X, Zhao B, Zheng HJ, Hu Y, Lu G, Yang CQ, Chen JD, Chen JJ, Chen DY, Zhang $L$, et al. Gossypium barbadense genome sequence provides insight into the evolution of extra-long staple fiber and specialized metabolites. Sci Rep. 2015:5:14139.

33. Yuan DJ, Tang ZH, Wang MJ, Gao WH, Tu LL, Jin X, Chen LL, He YH, Zhang $L$, Zhu LF, et al. The genome sequence of Sea-Island cotton (Gossypium barbadense) provides insights into the allopolyploidization and development of superior spinnable fibres. Sci Rep. 2015;5:17662.

34. Li F, Fan G, Lu C, Xiao G, Zou C, Kohel RJ, Ma Z, Shang H, Ma X, Wu J, et al. Genome sequence of cultivated Upland cotton (Gossypium hirsutum TM-1) provides insights into genome evolution. Nat Biotech. 2015;33:524-30.

35. Zhang T, Hu Y, Jiang W, Fang L, Guan X, Chen J, Zhang J, Saski CA, Scheffler BE, Stelly DM, et al. Sequencing of allotetraploid cotton (Gossypium hirsutum L. acc. TM-1) provides a resource for fiber improvement. Nat Biotech. 2015:33:531-7.

36. Liu RZ, Ai NJ, Zhu XX, Liu FJ, Guo WZ, Zhang TZ. Genetic analysis of plant height using two immortalized populations of "CRI12 x J8891" in Gossypium hirsutum L. Euphytica. 2014;196:51-61.

37. Li CQ, Wang CB, Dong N, Wang XY, Zhao HH, Converse $R$, Xia Z, Wang $R$, Wang QL. QTL detection for node of first fruiting branch and its height in upland cotton (Gossypium hirsutum L.). Euphytica. 2012;188:441-51.

38. Li CQ, Wang XY, Dong N, Zhao HH, Xia Z, Wang R, Converse RL, Wang QL. QTL analysis for early-maturing traits in cotton using two upland cotton (Gossypium hirsutum L.) crosses. Breeding Sci. 2013;63:154-63.

39. Guo YF, McCarty JC, Jenkins JN, Saha S. QTLs for node of first fruiting branch in a cross of an upland cotton, Gossypium hirsutum L., cultivar with primitive accession Texas 701. Euphytica. 2008;163:113-22.

40. Fan SL, Yu SX, Song MZ, Yuan RH. Construction of molecular linkage map and QTL mapping for earliness in short-season cotton. Cotton Sci. 2006;18: 135-9.

41. Guo YF, McCarty J, Jenkins JN, An CF, Saha S. Genetic detection of node of first fruiting branch in crosses of a cultivar with two exotic accessions of upland cotton. Euphytica. 2009;166:317-29.

42. Li C, Song L, Zhao H, Xia Z, Jia Z, Wang X, Dong N, Wang Q. Quantitative trait loci mapping for plant architecture traits across two upland cotton populations using SSR markers. J Agr Sci. 2014;152:275-87.

43. Lacape JM, Gawrysiak G, Cao TV, Viot C, Llewellyn D, Liu SM, Jacobs J, Becker D, Barroso PAV, de Assuncao JH, et al. Mapping QTLs for traits related to phenology, morphology and yield components in an interspecific Gossypium hirsutum x G. barbadense cotton RIL population. Field Crop Res. 2013;144:256-67.

44. Lu H, Lin T, Klein J, Wang S, Qi J, Zhou Q, Sun J, Zhang Z, Weng Y, Huang S. QTL-seq identifies an early flowering QTL located near Flowering Locus T in cucumber. Theor Appl Genet. 2014;127:1491-9.

45. Maurer A, Draba V, Jiang $Y$, Schnaithmann F, Sharma R, Schumann E, Kilian B, Reif JC, Pillen K. Modelling the genetic architecture of flowering time control in barley through nested association mapping. BMC Genomics. 2015;16:290.

46. Hulse-Kemp AM, Lemm J, Plieske J, Ashrafi H, Buyyarapu R, Fang DD, Frelichowski J, Giband M, Hague S, Hinze LL, et al. Development of a $63 \mathrm{~K}$ SNP array for cotton and high-density mapping of intraspecific and interspecific populations of Gossypium spp. G3 (Bethesda). 2015;5:1187-209.

47. Zhang Z, Shang H, Shi Y, Huang L, Li J, Ge Q, Gong J, Liu A, Chen T, Wang $D$, et al. Construction of a high-density genetic map by specific locus amplified fragment sequencing (SLAF-seq) and its application to Quantitative Trait Loci (QTL) analysis for boll weight in upland cotton (Gossypium hirsutum.). BMC Plant Biol. 2016;16:79.

48. Blenda A, Fang DD, Rami JF, Garsmeur O, Luo F, Lacape JM. A high density consensus genetic map of tetraploid cotton that integrates multiple component maps through molecular marker redundancy check. Plos One. 2012;7:e45739.

49. Said JI, Song MZ, Wang HT, Lin ZX, Zhang XL, Fang DD, Zhang JF. A comparative meta-analysis of QTL between intraspecific Gossypium hirsutum and interspecific $G$. hirsutum $\times$ G. barbadense populations. Mol Genet Genomics. 2015;290:1003-25.

50. Yoshida N, Yanai Y, Chen LJ, Kato Y, Hiratsuka J, Miwa T, Sung ZR, Takahashi S. EMBRYONIC FLOWER2, a novel polycomb group protein homolog, mediates shoot development and flowering in Arabidopsis. Plant Cell. 2001; 13:2471-81. 
51. Luo M, Platten D, Chaudhury A, Peacock WJ, Dennis ES. Expression, imprinting, and evolution of rice homologs of the polycomb group genes. Molecular Plant. 2009;2:711-23.

52. Liu MS, Chen LFO, Lin CH, Lai YM, Huang JY, Sung ZR. Molecular and functional characterization of Broccoli EMBRYONIC FLOWER 2 genes. Plant Cell Physiol. 2012;53:1217-31.

53. Knapp SJ, Stroup WW, Ross WM. Exact confidence intervals for heritability on a progeny mean basis. Crop Sci. 1985;25:192-4.

54. Paterson $\mathrm{AH}$, Brubaker $\mathrm{CL}$, Wendel JF. A rapid method for extraction of cotton (Gossypium spp.) genomic DNA suitable for RFLP of PCR analysis. Plant Mol Biol Rep. 1993;11:122-7.

55. Andolfatto P, Davison D, Erezyilmaz D, Hu TT, Mast J, Sunayama-Morita T, Stern DL. Multiplexed shotgun genotyping for rapid and efficient genetic mapping. Genome Res. 2011;21:610-7.

56. Chen W, Yao JB, Chu L, Li Y, Guo XM, Zhang YS. The development of specific SNP markers for chromosome 14 in cotton using next-generation sequencing. Plant Breeding. 2014;133:256-61.

57. Li H, Durbin R. Fast and accurate short read alignment with BurrowsWheeler transform. Bioinformatics. 2009;25:1754-60.

58. Li H, Handsaker B, Wysoker A, Fennell T, Ruan J, Homer N, Marth G, Abecasis G, Durbin R, Proc GPD. The Sequence Alignment/Map format and SAMtools. Bioinformatics. 2009:25:2078-9.

59. McKenna A, Hanna M, Banks E, Sivachenko A, Cibulskis K, Kernytsky A, Garimella K, Altshuler D, Gabriel S, Daly M, et al. The Genome Analysis Toolkit: A MapReduce framework for analyzing next-generation DNA sequencing data. Genome Res. 2010;20:1297-303.

60. Liu DY, Ma CX, Hong WG, Huang L, Liu M, Liu H, Zeng HP, Deng DJ, Xin HG, Song J, et al. Construction and analysis of high-density linkage map using high-throughput sequencing data. Plos One. 2014;9:e98855.

61. McCouch SR, Chen XL, Panaud O, Temnykh S, Xu YB, Cho YG, Huang N, Ishii T, Blair M. Microsatellite marker development, mapping and applications in rice genetics and breeding. Plant Mol Biol. 1997;35:89-99.

\section{Submit your next manuscript to BioMed Central and we will help you at every step:}

- We accept pre-submission inquiries

- Our selector tool helps you to find the most relevant journal

- We provide round the clock customer support

- Convenient online submission

- Thorough peer review

- Inclusion in PubMed and all major indexing services

- Maximum visibility for your research

Submit your manuscript at www.biomedcentral.com/submit

) Biomed Central 